\title{
Solid State Photon-Counters for High Time Resolution Astrophysics (HTRA)
}

\author{
G. Bonanno' \\ INAF- Catania Astrophysical Observatory \\ Via S. Sofia, 78, 95123 Catania, Italy \\ E-mail: giovanni.bonannodoact.inaf.it
}

\section{S. Billotta ${ }^{2}$}

INAF- Catania Astrophysical Observatory

Via S. Sofia, 78, 95123 Catania, Italy

E-mail: sergio.billottaloact.inaf.it

Complete author's list: (M. Belluso, M.C. Timpanaro, A. Grillo, G. Occhipinti, G. Naletto, T. Occhipinti, E. Verroi, C. Barbieri)

\begin{abstract}
The needs to have detector systems capable to push the time tagging capabilities of each incoming photon toward the $10 \mathrm{ps}$ region, sustaining up to $1 \mathrm{GHz}$ count rates continuously for hours of uninterrupted acquisition have generated a new type of investigation on detectors and electronic front-end that can be able to satisfy these requirements. For example, thanks to such extremely high time resolution and stability, and to the photon flux at the output of the future 40-m class telescopes as the European Extremely Large Telescope (E-ELT), these new instruments could study second order correlation functions in the photon stream from celestial sources in their different manifestations (statistical characteristics of the photon stream, photon correlation spectroscopy, Hanbury-Brown-Twiss intensity interferometry over two or more apertures). To investigate the real possibilities of such devices, we studied and characterized different detectors produced by various manufacturers. Some results particularly focused on timing resolution and photo detection efficiency will be presented. Finally a brief discussion on recent developments in that field is also presented.
\end{abstract}

Keywords: high-speed photometry, high-speed detectors, detectors characterization, Single Photon Avalanche Diode (SPAD).

\footnotetext{
$1 \quad$ Speaker
} 


\begin{tabular}{ll}
\hline PSS & PROCEEDINGS \\
OF SCIENCE
\end{tabular}

High Time Resolution Astrophysics IV - The Era of Extremely Large Telescopes-HTRA-IV Agios Nikolaos, Crete, Greece

May 5-7 2010 


\section{Introduction}

During these last years the study of fast transient phenomena with high time resolution has progressively gained a role of a certain importance. Many scientific cases have been proposed requiring fast and very fast response detectors. Thus the need to have detectors able to push the time tagging capabilities of each incoming photon toward the tenth of picoseconds region, sustaining up to $1 \mathrm{GHz}$ count rates continuously for hours of uninterrupted acquisition has generated an investigation on detectors and electronic front-end that can be able to satisfy these requirements. High time resolution and very accurate time tagging will be surely exploited by the future 40-m class telescopes as the European Extremely Large Telescope (E-ELT). Second order correlation functions in the photon stream from celestial sources in their different manifestations will be accessible as well as the Hanbury-Brown-Twiss intensity interferometry over two or more apertures. In this paper we reviewed and compared some current available detector systems considering of crucial importance the detector fast response. Great attention has been done to relatively new solid state devices named Single Photon Avalanche Diode (SPAD), that are very promising in showing excellent timing resolution and sensitivity. To investigate the real possibilities of such devices, we studied and characterized different detectors produced by various manufacturers. In section 3 we present some results particularly focused on timing resolution and photo detection efficiency (PDE). In section 4 we briefly describe the IquEYE instrument that represents a good application of SPAD detectors. In the same section we pointed out how we conceived the front-end electronics to be easily expanded when SPAD arrays will be available. Finally, to better understand what can be expected in the near future, we compared in section 5 the two currently available technologies used to manufacture this kind of devices.

\section{Scientific motivations and possible instrument for picoseconds time-scale observations}

As well known in astrophysics the term "High Time Resolution Astrophysics" (HTRA) is used to refer to scientific applications requiring fast detectors (with high sensitivity), fast readout electronics and fast data acquisition. But we have to specialize the term as regards the time-scale of observation. For scientific applications like stellar flares and pulsations or close binary systems, time-scales of the order of milliseconds could be sufficient, while for observations of stellar oscillations (white dwarfs or neutron star) time-scales of the order of microseconds will be necessary. Other scientific projects like observation of pulsars or "Intensity Interferometry" require sub-microseconds time scales and accurate time tagging and thus require detectors systems capable to operate in photon counting and with time tagging capabilities of each incoming photon toward the picoseconds region, sustaining up to $1 \mathrm{GHz}$ count rates continuously for hours of uninterrupted acquisition [1]. We named the instruments able to satisfy these requirements "Quantum-Photometers". 
Such instruments applied to extreme large telescopes, thanks to the much higher photon flux, to the extremely high timing accuracy and to the very long term stability could allow the study of stellar objects with time resolution better than one or two order of magnitude of the current [2].

Here we compare some current available detector systems in terms of fast response, photon counting and time tagging capability:

- MCPs + CCD or CMOS-APS

A photon counting imaging array detector placed at the telescope focal plane would be the simplest and easiest way to operate. A detector that make use of a CCD (or APS) coupled with a Micro Channel Plate (MCP) takes milliseconds to acquire the entire image and thus only a count rate of $\mathrm{KHz}$ can be obtainable and a photon time tagging is impossible to arrange. Furthermore the MCP photocathode show a relatively low PDE in the visible range.

- EMCCD

This imaging array has the advantage respect to the MCP+CCD to have the electron multiplication on chip and thus each pixel can show the typical high photon detection efficiency of a back-illuminated CCD. This kind of detector can operate at frequencies of the order of few KHz. Improvements in timing resolution could be obtainable by adding further readout ports. In that case a time resolution of hundreds of microseconds could be feasible, but a precise time tagging is practically impossible.

- SPAD array

This solid state device in the array format is, at the moment, a non-imaging detector due to the considerable interspacing between pixels. To overcome this problem appropriate optical fibers and microlenses are under development. Figure 1 shows a layout of a $5 \times 5$ pixels array with each pixel having a diameter of $40 \mu \mathrm{m}$. The interspacing between pixels is $240 \mu \mathrm{m}$.

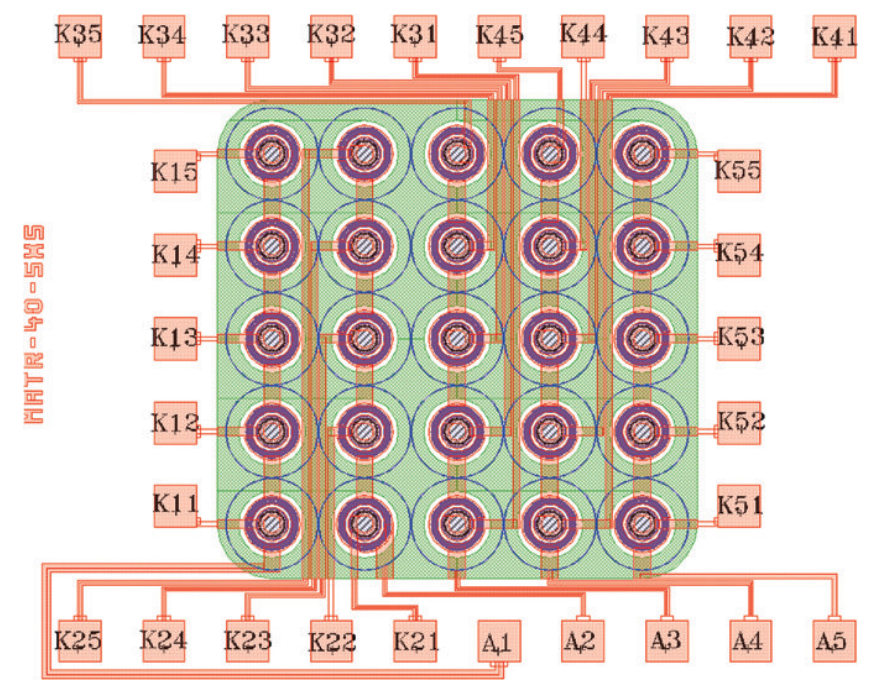

Figure 1: Plane view of a $5 \times 5$ SPADs array layout fabricated by STMicroelectronics. Each pixel has a diameter of $40 \mu \mathrm{m}$ and the interspacing between pixels is $240 \mu \mathrm{m}$. 
SPADs are semiconductor junction diodes reverse-biased a few Volts above the breakdown voltage. At this operating condition, the electric field within SPAD depletion layer is so high that a single carrier injected in this region can trigger a self- sustaining avalanche multiplication process. As a result, a sharp current pulse of few milliamps and with sub-nanosecond rise time is produced. If the first carrier is photo-generated, the current rising edge marks the photon arrival time. Once the breakdown current has been detected, it is quenched by a resistor (passive quenching) or by a quenching circuit (active quenching). The diode is thus turned off for a suitable hold-off time that allows to dissipate the charges stored within the depletion layer. After this time, the voltage is restored to the bias value and the device is ready to detect another photon [3].

The use of a single SPAD element has two adverse consequences respect to the array:

- $\quad$ finding an optical solution coping with very small sensitive areas

- fixed aperture photometer, without imaging capability, sets limitations to a very good scientific utilization.

Thus the baseline solution for an instrument capable of fast and accurate time tagging could be a non-imaging photometer made by a focal reducer plus a $32 \times 32$ lenslet array that fed through a bundle of fibers a $32 \times 32$ pixel detector.

\section{Characteristics of current available most performing detectorfor fast and accurate time tagging applications}

As said in the previous section, at the moment the technology is not mature to deliver suitable SPAD arrays for HTRA applications but is very promising. To evaluate the real performance of such devices at the telescope we decided to use single SPAD as preliminary step and prepare all the necessary for the future availability of arrays. In order to select a suitable device for our project we characterized different SPAD detectors produced by various manufacturers. The relevant characteristics of these devices to be used as HTRA detectors are essentially the time tagging accuracy, the photo detection efficiency (PDE) and the dynamic range.

As can be seen in the next sub-sections SPADs have good characteristics:

- time tagging accuracy better than $100 \mathrm{ps;}$

- PDE greater than $50 \%$ in the visible;

- dynamic range greater than $100 \mathrm{Mc} / \mathrm{s}$.

\subsection{Time tagging accuracy}

The time resolution characterization has been carried out using a time-correlated single photon counting apparatus whose schematic diagram is shown in Figure 2. The apparatus is constituted essentially by a pulsed laser system that has a pulse width of $40 \mathrm{ps,} \mathrm{a} \mathrm{multiple} \mathrm{grey}$ filter to reduce its intensity at will, and a time-to-amplitude converter (10 ps resolution) to build the distribution of the time intervals between the laser trigger and the photon detection on the SPAD [4]. 


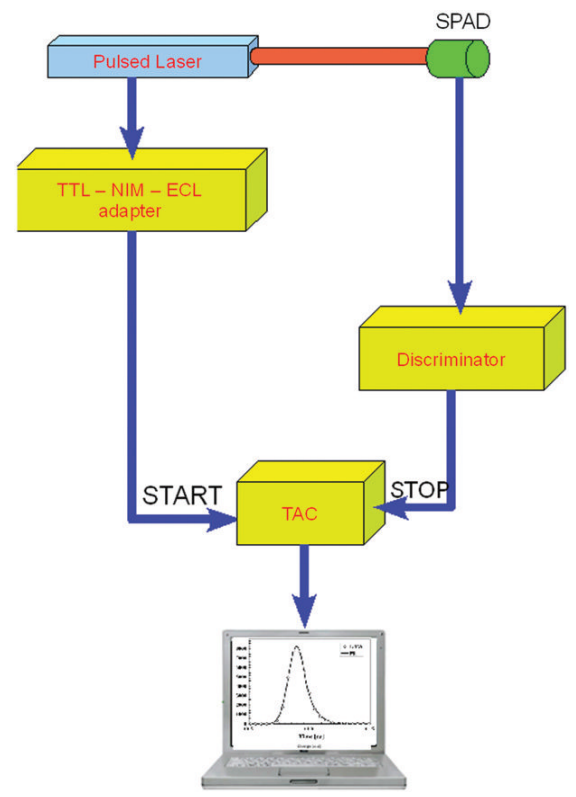

Figure 2: Scheme of the apparatus used for time resolution measurements. A pulsed laser system equipped with a multiple grey filter illuminates a SPAD, at the same time the trigger from the laser sends the START to a time-to-amplitude converter (TAC). When the SPAD detects the photon from the laser it sends the STOP to the TAC.

From the measurements we found a reconstruction of the laser pulse time structure, which confirms the validity of the method, and that a time accuracy of better than 40 ps has been achieved.

\subsection{Photon Detection Efficiency (PDE)}

The equipment used for PDE characterization is one of the available facilities at INAF Osservatorio Astrofisico di Catania laboratory. Figure 3 shows a scheme of the experimental set up [5] and here the implemented main parts are briefly presented.

From right to left you can find: a Xenon lamp used as the radiation source, a wavelength selection system constituted essentially by a set of filters and a Czerny-Turner monochromator a beam splitter to direct the monochromatic radiation towards an integrating sphere that hosts a NIST traced reference detector and the SPAD. The use of an integrating sphere is crucial because the very small size of the SPAD with respect to the optical beam. 


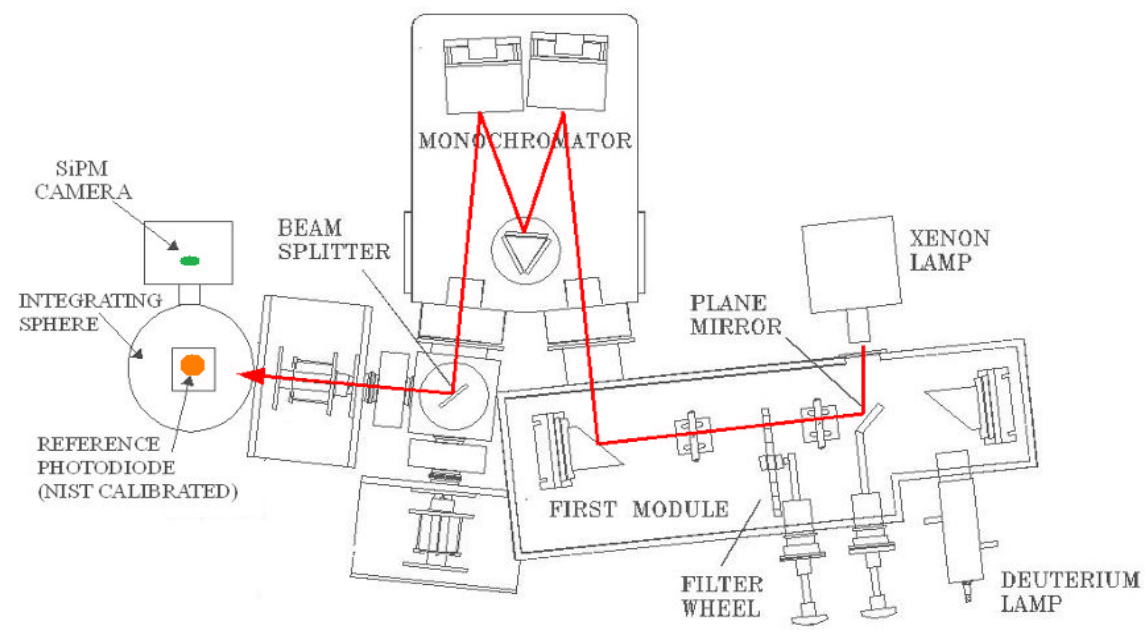

Figure 3: Scheme of the implemented mechanical and optical parts of the apparatus to characterize the SPAD PDE. The red line indicates the light path. A Xenon lamp is used as the radiation source, several filters and mirrors are used to obtain a bandwidth around the wavelength of interest and to focus the beam into the monochromator, which allows to select a line in the 130-1100 nm spectral range with a FWHM better than $1 \mathrm{~nm}$. The monochromatic light is directed by means of a beam splitter and focused through a lens inside an integrating sphere, in order to produce an uniform light.

In Figure 4 the resulting measured PDE for a SPAD manufactured by MPD and utilized by the AquEYE and IquEYE photometers is shown [4]. As can be clearly noted the PDE approaches $60 \%$ at $550 \mathrm{~nm}$.

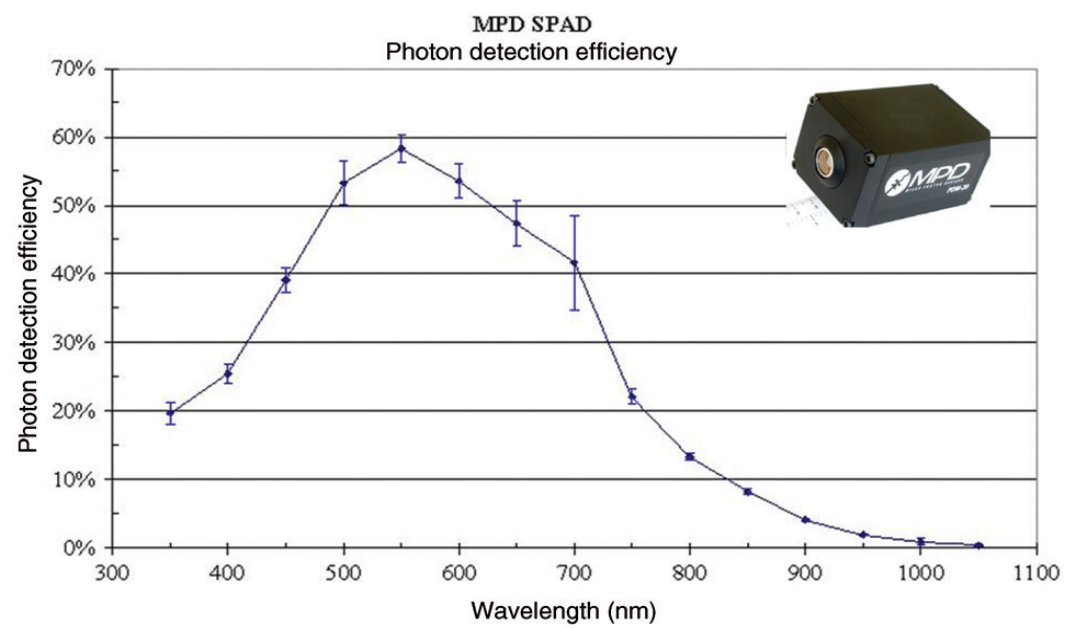

Figure 4: Photon detection efficiency of one of the MPD SPADs utilized for the AquEYE and IquEYE instruments. This device shows a PDE peak of about $60 \%$ at $550 \mathrm{~nm}$ 


\subsection{Dynamic range and after-pulsing}

By using the set-up apparatus described in section 3.2 and by means of several neutral filters or changing the aperture of the entrance or exit slits of the monochromator we can vary the photon flux intensity coming into the integrating sphere and then obtain measurements of counts linearity and dynamic range [4]. While the measurement of the afterpulse effects, is achieved by measuring, at different over-voltages the dark count rate varying the hold off time by means of the AQC whose schematic diagram is shown in Figure 5.

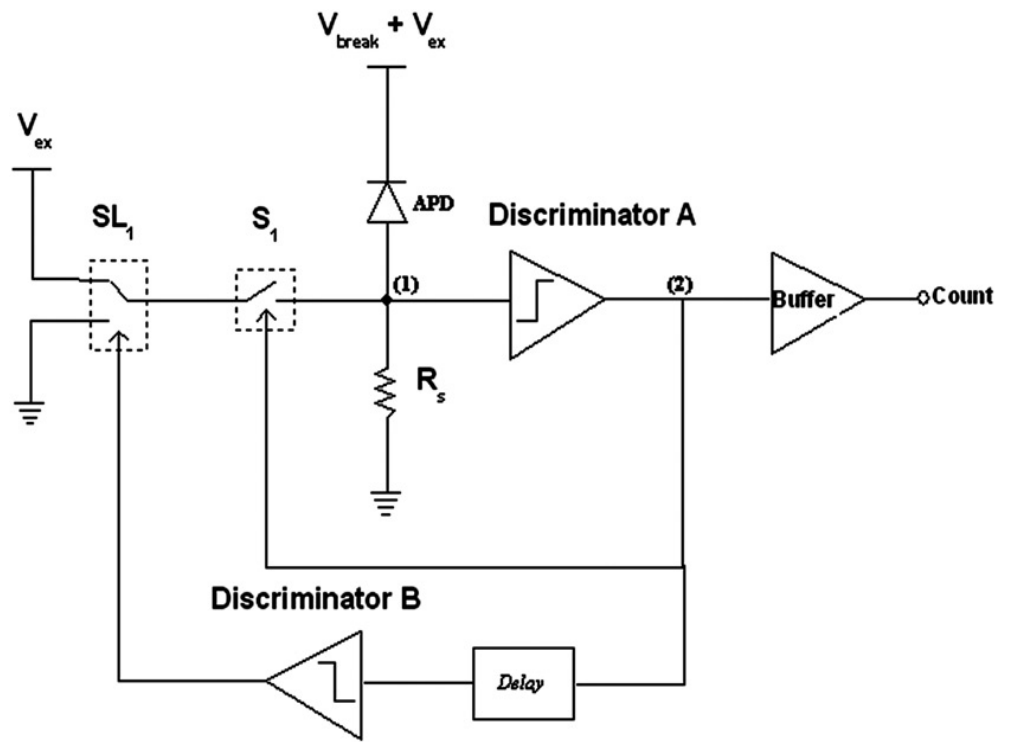

Figure 5: Schematic of an Active Quenching Circuit. This circuit is able to bias the SPAD, provides for extinguishing the avalanche after the firing, bringing the SPAD to its waiting conditions and after a changeable hold-off time making the SPAD ready to detect another photon.

From our measurements we essentially found the characteristics reported on the manufacturer's MPD data sheet:

- a dead time of $75 \mathrm{~ns}$ after each detected event;

- a typical after-pulsing probability around $1 \%$;

- a linear count rate up to $2 \mathrm{MHz}$ (NIM output) or $12 \mathrm{MHz}$ (TTL output);

- a dark count rate less than $50 \mathrm{cnts} / \mathrm{s}$.

\section{Examples of SPAD application for HTRA: AquEYE and IquEYE}

A very good example of implementation of these type of devices in astronomical instruments for HTRA, is constituted by the two photometers AquEYE [6]and IquEYE [7], [8] designed and realized by a group led by prof. Cesare Barbieri from Padua University. Each instrument, is conceptually a simple fixed aperture photometer which collects the light within a 
FoV of just a few arcseconds (selectable from 1" to 6"), splits the telescope light beam in four equal parts, and focuses each sub-beam on an independent SPAD. A detailed description of the instruments and the achieved scientific results is treated by $\mathrm{C}$. Barbieri in these proceedings.

As discussed in the Barbieri's paper (these proceedings), the Data Acquisition System that collects the pulses produced by each SPAD for every detected photon is based on of a time to digital converter (TDC) board (CAEN V1290N, see Figure 6). This TDC board is able to timetag the pulses present on the 16-channels inputs with a 24.4 ps time resolution.
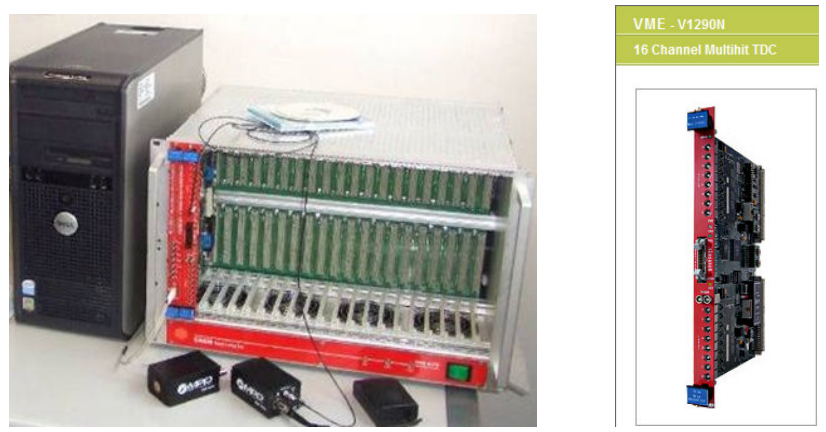

Figure 6: CAEN VME crate hosting various VME boards (left) and the CAEN V1290N board that allows to treat 16-channels Time to Digital Converter (TDC) simultaneously (right).

To emphasize the easily expandability of our data acquisition system, we here briefly describe the front-end electronics, conceived to drive also SPAD arrays when they will be ready with adequate parameters. By using a VME crate we can use "standard" VME boards produced by CAEN and we can easily add the necessary boards. The rest (PC, interface boards, etc.) will remain the same with few changes on the software procedures. The system, actually, with a $\mathrm{V} 1290 \mathrm{~N}$ board is able to acquire the events from 16 channels (for example from an array of $4 \times 4$ SPADs). If an array of $32 \times 32$ pixels will be available from manufacturers in the near future we can simply use four V1190A VME boards, each board allowing 128 channels TDC for a total of 1024 channels as shown in Figure 7.
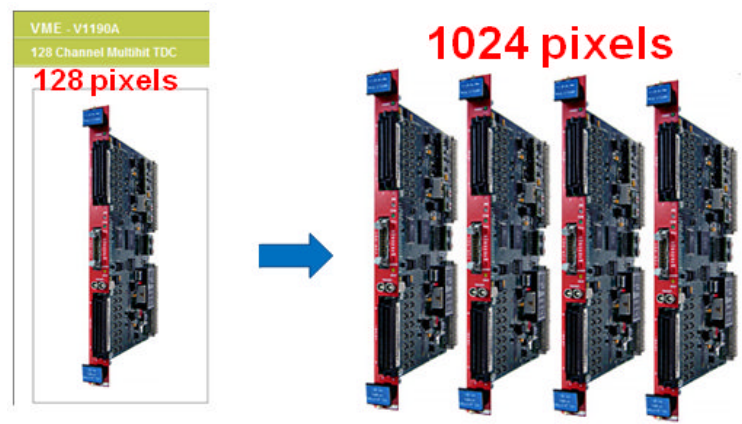

Figure 7: CAEN V1190A board that allows to treat 128-channels TDC simultaneously. Four V1190A boards can take place easily on the VME crate allowing to acquire events from $32 \times 32$ pixels SPAD array. 
Thus we can conclude that both instruments can be considered as precursors of high density SPAD detectors for HTRA observations to be used for extreme large telescopes or for Hanbury-Brown and Twiss intensity interferometry.

The intense use of this kind of instrument and the continuous experience that we can gain from the utilization and improvements of the electronics can give us very useful information to implement thousands of TDC directly on chip or specialized FPGA-ASIC circuits.

\section{Near Future: from single pixel to array}

As explained in section 2, the need to have SPAD arrays capable of imaging, photoncounting and time tagging is considerable, and some SPAD manufactured and university laboratories around the world have pursued the objective to produce good quality SPAD arrays. The Politecnico di Milano (POLIMI) has a long experience in the field. Recently the group has developed a $32 \times 32$ pixel array in CMOS technology [9]. By using the CMOS technology the POLIMI group has successfully integrated on chip the quenching circuit, the counter and the readout electronics for each pixel. Figure 8 shows on the left the array layout and on the right the pixel layout. Each pixel has a sensitive area of $20 \mu \mathrm{m}$ diameter while the total area occupied by the pixel and electronics is $100 \mu \mathrm{m}$ and thus a fill factor of $20 \%$ is obtained. This means that this device is not a true imaging device and need specialized optics (optical fibers or microlenses) to recover the fill-factor problem.
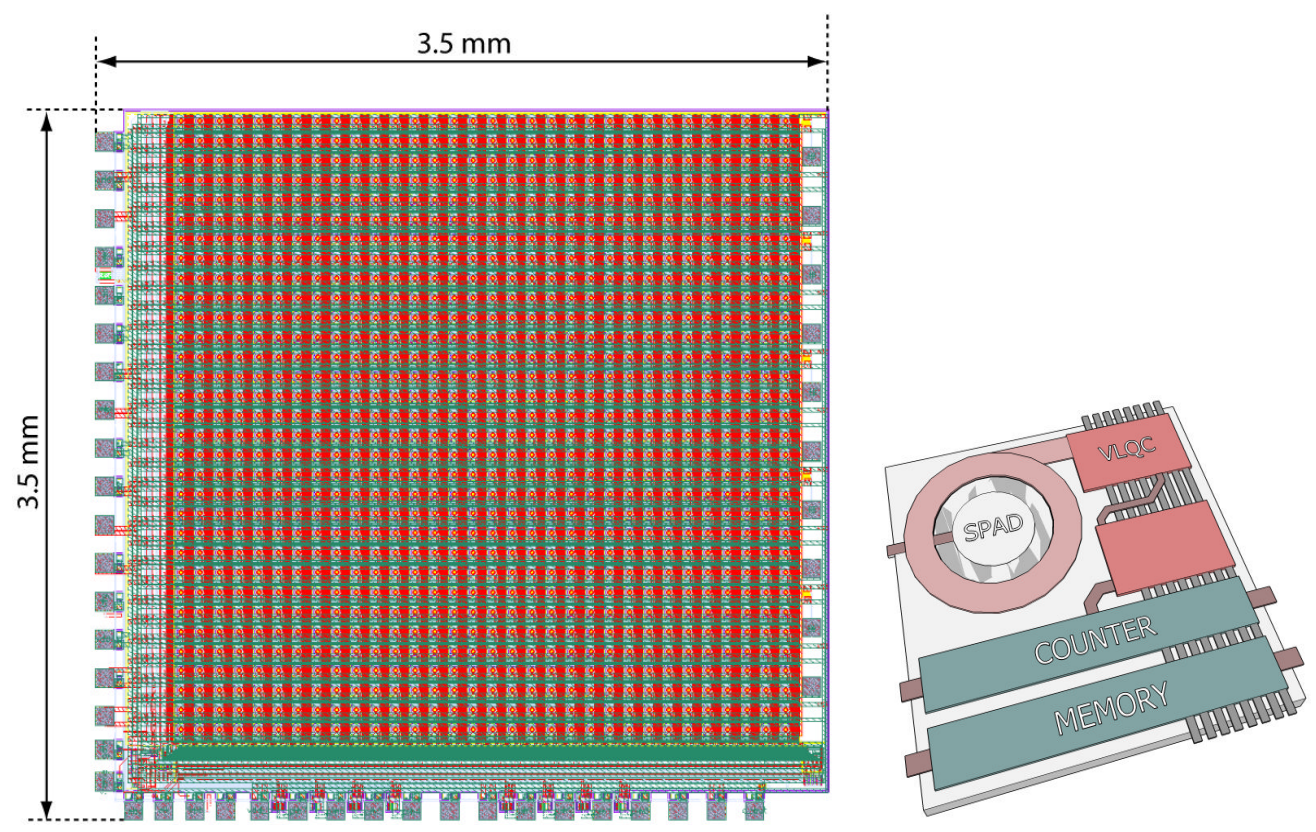

Figure 8: $32 \times 32$ SPAD array layout (left). Pixel layout (right). The array has been manufactured by POLIMI. Each pixel has a sensitive area of $20 \mu \mathrm{m}$ diameter while the total area including the ancillary electronics is $100 \mu \mathrm{m}$. 
The relevant characteristics of this detector are:

- Up to 100,000 frames/s for the whole image meaning $20 \mu$ s time tagging

- 1024 single photon pixels

- Negligible inter-frame dead-time (20 ns)

- Multi-photon detection capability

- CMOS technology

The technology is good to have a very compact detector array system (integrating on chip all the necessary electronics) but, unfortunately, shows a poor PDE in the Near-IR band, as will be seen in the next section.

\section{Comparison between current available SPAD technologies}

Two technologies are currently used to produce SPAD detectors:

- the classical one that is referred to us as "Planar epitaxial"

- the recent developed $0.35 \mu \mathrm{m}$ high-voltage CMOS technology [9]

The two technologies are quite different and while the first not allow to integrate driving electronics on chip the second, unfortunately have the critical point that the sensitive area is very thin. In Figure 9, that shows the cross-section of a planar epitaxial SPAD (left) and of a CMOS-SPAD (right), the thickness of the sensitive area where the photon impinges for the planar epitaxial SPAD is thicker than that for the CMOS SPAD.
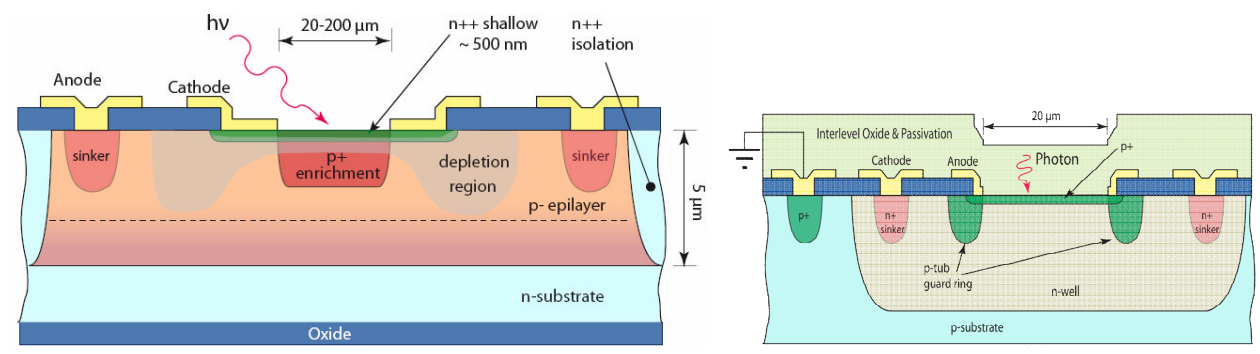

Figure 9: On the left is shown the cross-section of a "Planar epitaxial" SPAD while on the right is shown the cross-section of a SPAD fabricated with the $0.35 \mu \mathrm{m}$ highvoltage CMOS technology. The thickness of the sensitive area of the "Planar epitaxial" SPAD is thicker than that of the CMOS SPAD.

The sensitive area thickness is the cause of the low PDE values at wavelengths greater than $650 \mathrm{~nm}$ as shown in the PDE plot of Figure 10. In fact the absorption length in Silicon depends on the thickness and on the radiation wavelength. At wavelengths greater than $650 \mathrm{~nm}$ the absorption length is greater than about $4 \mu \mathrm{m}$ and thus the detector becomes "transparent" at these wavelengths.

The epitaxial technology is well consolidated and allows to obtain high PDE values as demonstrated in section 3.2. 


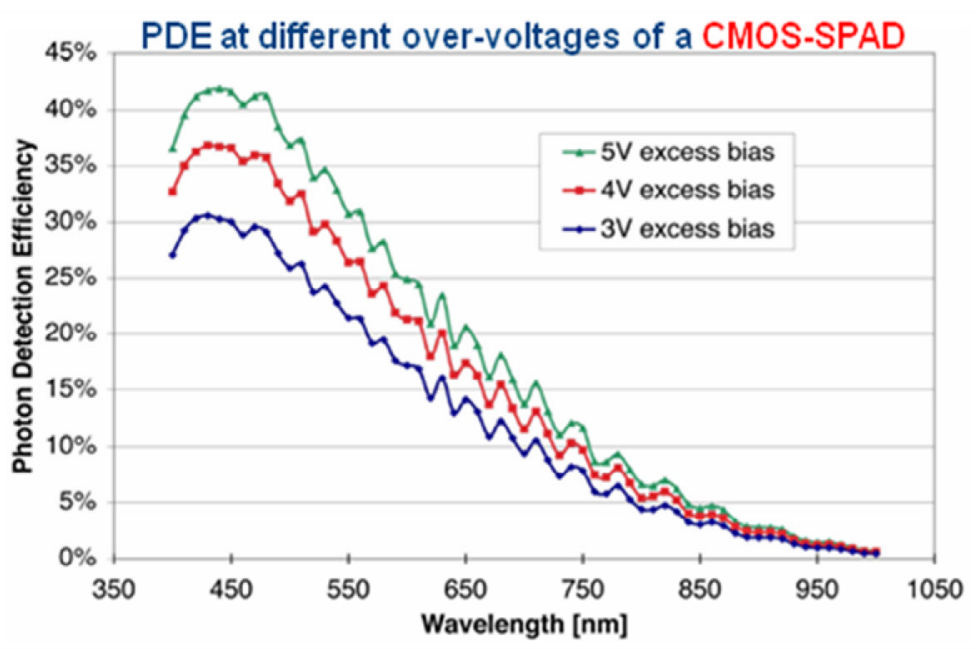

Figure 10: PDE at different over-voltages of a POLIMI CMOS-SPAD. The low PDE values obtained at wavelength down $650 \mathrm{~nm}$ are due to the thickness of the sensitive area.

Fortunately both technologies are not at the final stage and further improvements are in progress. To increase the PDE in the NIR and IR bands the CMOS technology has to complain with the thickness of the sensitive area. The epitaxial technology shows a reasonable PDE in the blue and visible bands but fails in having SPAD arrays with more than thousands pixels and with low dark counts for each pixel even at temperatures as low as $-20{ }^{\circ} \mathrm{C}$. In fact at the moment the limiting factor of this technology to go in the production of high density arrays is the controlling of dark count rates [4], [10].

\section{Conclusions}

HTRA can rely on extremely fast photon counting photometers utilizing new solid state detectors and appropriate electronics capable of accurate time tagging better than $1 \mathrm{~ns}$.

These detectors, are characterized by a fast time response of hundreds of ps, a PDE that approaches $60 \%$ in the visible, a very low dark count rate and a good linearity and thus can be considered good candidate for applications requiring high time resolution and accurate time tagging.

Time-resolved measurements have been demonstrated by Iqueye, a fast photometer based on these detectors, mounted at the ESO-NTT focal plane (see C. Barbieri this procedings).

The state of the art of SPAD technology is currently unable to deliver two dimensional arrays because the poor PDE for the CMOS case and because the fabrication problems for the planar epitaxial case.

Some technology developments are in progress and we are confident that soon photon arrays with good performance in terms of PDE, dark counts and time tagging will be available for very-fast and accurate imaging photometry. In this framework a front-end electronics and data acquisition that can be easily expanded certainly helps in following this progress. 


\section{References}

[1] C. Barbieri, G. Naletto, T. Occhipinti, C. Facchinetti, E. Verroi, E. Giro, A. Di Paola, S. Billotta, P. Zoccarato, P. Bolli, F. Tamburini, G. Bonanno, M. D’onofrio, S. Marchi, G. Anzolin, I. Capraro, F. Messina, M. Belluso, C. Pernechele, M. Zaccariotto, L. Zampieri, V. Da Deppo, S. Fornasier, F. Pedichini, AquEYE, a single photon counting photometer for astronomy, Journal of Modern Optics, 56(2), 261-272, 2009.

[2] D. Dravins, C. Barbieri, V. Da Deppo et al., QuantEYE quantum optics instrumentation for astronomy. OWL Instrument Concept Study, Tech. rep., ESO, Document OWL-CSR-ESO-000000162, 2005.

[3] M. Mazzillo, A. Piazza, G. Condorelli, D. Sanfilippo, G. Fallica, S. Billotta, M. Belluso, G. Bonanno, L. Cosentino, A. Pappalardo, P. Finocchiaro, Quantum Detection Efficiency in Geiger Mode Avalanche Photodiodes, IEEE Transactions on Nuclear Science, 55, (6), 3620 -3625, 2008.

[4] S. Billotta, C. Barbieri, M. Belluso, G. Bonanno, G. Condorelli, S. Di Mauro, P.G. Fallica, P. Finocchiaro, M. Mazzillo, A. Pappalardo, D. Sanfilippo, C. Timpanaro, Characterization of Detectors for the Italian Astronomical Quantum Photometer Project, Journal of Modern Optics. 56(2), 273-283, 2008.

[5] G. Bonanno, P. Bruno, A. Calì, R. Cosentino, R. Di Benedetto, M. Puleo, S. Scuderi, The Catania Astrophysical Observatory facility for UV CCD characterization, SPIE Proceedings, Vol. 2808, p. 242, 1996.

[6] C. Barbieri, G. Naletto, T. Occhipinti, C. Facchinetti, E. Verroi, E. Giro, A. Di Paola, S. Billotta, P. Zoccarato, P. Bolli, F. Tamburini, G. Bonanno, M. D'onofrio, S. Marchi, G. Anzolin, I. Capraro, F. Messina, M. Belluso, C. Pernechele, M. Zaccariotto, L. Zampieri, V. Da Deppo, S. Fornasier, F. Pedichini, AquEYE, a single photon counting photometer for astronomy, Journal of Modern Optics, 56(2), 261-272, 2009.

[7] C. Barbieri, G. Naletto, I. Capraro, T. Occhipinti, E. Verroi, P. Zoccarato, C. Facchinetti, C. Germanà, M. Parrozzani, M. Zaccariotto, G. Anzolin, F. Tamburini, A. Di Paola, E. Giro, G. Bonanno, S. Billotta, C. Pernechele, P. Bolli, L. Zampieri, A. Possenti, A. Cadez, Very fast photon counting photometers for astronomical applications: IquEYE for the ESO $3.5 \mathrm{~m}$ New Technology Telescope, SPIE Conference 7355B on Photon Counting Applications, paper number: 7355-25, Prague, 2009.

[8] G. Naletto, C. Barbieri, T. Occhipinti, I. Capraro, A. Di Paola, C. Facchinetti, E. Verroi, P. Zoccarato, G. Anzolin, M. Belluso, and 13 coauthors (2009): Iqueye, a single photon-counting photometer applied to the ESO new technology telescope, Astronomy and Astrophysics, 508(1), 531-539, 2009.

[9] F. Guerrieri, S. Tisa, F. Zappa, Fast Single-Photon Imager acquires 1024 pixels at $100 \mathrm{kframe} / \mathrm{s}$, Proc. SPIE, Vol.7249, doi: 10.1117/12.807426, 2009.

[10] Zappa, F., Tisa, S., Cova, S., Maccagnani, P., Saletti, R., Roncella, R, Bonaccini Calia, D.,Silber, A., Bonanno, G., Belluso, M., "Single Photon Avalanche Diodes Arrays for Astrophysics", Journal of Modern Optics, vol. 54; p. 163-189, 2007. 